\title{
Gut Microbiota Structure of Dead Migratory Birds in Dali Nouer Lake, Chifeng City, China, based on Bacterial 16S rRNA Amplicon Sequencing
}

\section{Lin Zheng}

Jilin Agricultural University

\section{Ling-Wei Zhu}

Changchun Veterinary Research Institute, Chinese Academy of Agricultural Sciences/Key laboratory of Jilin Province for Zoonosis Prevention and Control

\section{Ming-Wei liu}

Jilin Agricultural University

\section{Jia-yao Guan}

Changchun Veterinary Research Institute, Chinese Academy of Agricultural Sciences/Key laboratory of Jilin Province for Zoonosis Prevention and Control

\section{Lin-Hong Xie}

Changchun Veterinary Research Institute, Chinese Academy of Agricultural Sciences/Key laboratory of Jilin Province for Zoonosis Prevention and Control

\section{Jie Jing}

Changchun Veterinary Research Institute, Chinese Academy of Agricultural Sciences/Key laboratory of Jilin Province for Zoonosis Prevention and Control

\section{Ge-Jin Lu}

Changchun Veterinary Research Institute, Chinese Academy of Agricultural Sciences/Key laboratory of Jilin Province for Zoonosis Prevention and Control

\section{Xue Ji}

Changchun Veterinary Research Institute, Chinese Academy of Agricultural Sciences/Key laboratory of Jilin Province for Zoonosis Prevention and Control

\section{Dong Chu}

Changchun Veterinary Research Institute, Chinese Academy of Agricultural Sciences/Key laboratory of Jilin Province for Zoonosis Prevention and Control

\section{Xue-Jun Guo}

Changchun Veterinary Research Institute, Chinese Academy of Agricultural Sciences/Key laboratory of Jilin Province for Zoonosis Prevention and Control

Ping Chen (D 1243377523@qq.com )

Jilin Agricultural University

\section{Yang Sun}


Changchun Veterinary Research Institute, Chinese Academy of Agricultural Sciences/Key laboratory of Jilin Province for Zoonosis Prevention and Control

\section{Research Article}

Keywords: migratory birds, $16 \mathrm{~S}$ rRNA amplicon, gut microbiota

Posted Date: October 15th, 2021

DOl: https://doi.org/10.21203/rs.3.rs-948511/v1

License: (c) (i) This work is licensed under a Creative Commons Attribution 4.0 International License. Read Full License 
1 Gut microbiota structure of dead migratory birds in Dali Nouer Lake, Chifeng City, China, based on bacterial 16S rRNA

Lin Zheng ${ }^{1,2 * a}$, Ling-Wei Zhu ${ }^{2 * a}$, Ming-Wei liu ${ }^{1,2}$, Jia-yao Guan², Lin-Hong Xie ${ }^{3}$, Jie Jing ${ }^{2}$, Ge-Jin

${ }^{1}$ School of Food and Engineering, Jilin Agricultural University, Changchun, China;

${ }^{2}$ Changchun Veterinary Research Institute, Chinese Academy of Agricultural Sciences/Key

laboratory of Jilin Province for Zoonosis Prevention and Control, Changchun, China;

${ }^{3}$ State Forestry Administration, Shenyang, China

*a The authors contribute equally

*b Corresponding author. School of Food and Engineering, Jilin Agricultural University, Changchun 130118, China.

E-mail address: 1243377523@qq.com ( Ping Chen )

${ }^{* b}$ Corresponding author. Key laboratory of Jilin Province for Zoonosis Prevention and Control, Changchun 130122, China.

E-mail address: xuejung@yahoo.com (Xue-junGuo )

In August 2018, hundreds of migratory birds died in the area of Dali Nouer Lake, Chifeng City, China. We collected the remains of dead birds along with water and aquatic plants from the birds' 
environment. The bacterial communities of all samples were profiled by high-throughput sequencing of the V3-V4 hypervariable region of the 16S rRNA amplicon. At the genus level, Bacteroides, Clostridium, Plesiomonas, Vibrio, Fusobacterium, and Aeromonas were the dominant genera in dead birds, the lake water, and aquatic plants in 2018. However, the relative abundances of these bacterial genera were significantly reduced compared with the levels obtained from healthy migratory bird feces, lake water, and aquatic plants from the same place and time period in 2019. Combined with environmental factors such as the changes in salt content and $\mathrm{pH}$, the invasion and reproduction of those pathogens may have promoted the decline and death of the birds.

Keywords: migratory birds, 16S rRNA amplicon, gut microbiota

\section{Introduction}

As migratory birds can carry and spread pathogenic microorganisms over long distances, this has raised international awareness of their health problems (Dijk et al., 2018; Allarda et al., 2019, Alvarez et al., 2010). In August 2018, hundreds of migratory birds died at Dali Nouer Lake of Chifeng City, Inner Mongolia autonomous region, China. Among the species of birds were Larus ridibundus, Pluvialis squatarola, Tadorna ferrginea, Anas poecilorhyncha, Aix galericulata, and Tadorna ferrginea. The dead birds were found to have congestion or bleeding in the intestinal mucosa or an enlarged liver and pancreas, but no obvious abnormalities were observed in other visceral organs. Upon first observation, some birds had no obvious clinical lesions, only displaying signs of malnutrition and wasting. To prevent a pandemic of zoonotic bacteria, attention should be paid to the bacterial flora carried by migratory birds, especially during outbreaks. It is known that interactions between animals and their gut microbiota play an important role in regulating host physiological processes (Round \& Mazmanian, 2009; Sommer \& Bäckhed, 2013). The host's gut microbiota will be altered when external pressures change (Zaneveld, McMinds, \& Vega, 2017). We collected the remains of the dead birds along with samples of water and aquatic plants from the lake to examine the structure of the microbiota and the presence of pathogens. Because there were no relevant data before the present pandemic, in the following year we collected and analyzed the stools of healthy migratory birds (no outbreak) and the same environmental samples mentioned above from the same locations as the references.

\section{Materials and Methods}

\section{Sample collection and preparation}


In 2018, 19 freshly dead migratory birds (not corrupted) were collected at Dali Nouer Lake of Chifeng City, Inner Mongolia autonomous region, China. Their visceral organs and intestinal tracts were sampled. Meanwhile, lake water and aquatic plant samples were also collected from three different points; two were from Dali Nuoer Lake (Nanhekou and Beihekou) where dead birds were found, and the third was from the contiguous Ganggeng Nuover Lake without dead birds. Based on morphological observations, we assigned lesion samples: intestinal tract (A3C, A5C, A8C, A11C, A12C, A13C, A14C, A18C, A19C) and visceral organs (A2G, A2F, A3F, A5G, A5F, A8G, A8F, A11G, A11F, A13G, A13F, A13Y, A14G) (Table 1).

In 2019, we also collected stool samples from healthy migratory birds, lake water, and aquatic plants from the same points (Figure 1). The host species of feces were determined by molecular methods (Verma et al., 2003), and 10 feces samples from identical hosts were mixed into one for sequencing. The detailed information of all samples is listed in Table 1.

The $\mathrm{pH}$ of lake water was determined using a $\mathrm{pH}$ meter, and salinity was determined using a conductivity meter. Meanwhile, $100 \mu \mathrm{L}$ water samples were diluted into three concentrations using physiological saline, then spread on Brain-Heart-Agar-Medium, and incubated at $37^{\circ} \mathrm{C}$ overnight. Finally, single colonies (CFU) on the agar plates were counted. After all indicators were tested, the $500 \mathrm{~mL}$ water samples were concentrated to $50 \mathrm{~mL}$ and stored at $-80^{\circ} \mathrm{C}$ until DNA extraction.

\section{Extraction of genomic cDNA and 16s rRNA sequencing}

The genomic DNA was extracted using different DNA kits (Omega Biotek) according to the sample characteristics. DNA was amplificated using paired 341F/806R primers that am plified a $425 \mathrm{bp} \mathrm{V3-V4} \mathrm{region} \mathrm{of} \mathrm{the} \mathrm{16S} \mathrm{rRNA} \mathrm{bacterial} \mathrm{gene,} \mathrm{and} \mathrm{amplicons} \mathrm{were} \mathrm{the}$ n sequenced using an Illumina HiSeq PE250 platform (Caporaso et al., 2012; the full prot ocol for these primers is available at www.bioplatforms.com).

\section{Sequence processing}

The effective sequences were clustered into operational taxonomic units (OTUs) with Usearch using a 97\% identity threshold. Before downstream analysis, all samples were randomly re-sampled at the minimum depth of all samples to minimize the bias of unequal sequencing depth. Taxonomic information of each OTU was assigned using the Ribosomal Database Project classifier (Version 2.2) after classification according to the number of sequences in each OTU.

Sequences were clustered followed by chimera filtering. In using OTUs for species classification, each OTU was considered to represent one species. We chose representative 
sequences for each OTU and used the RDP (http://rdp.cme.msu.edu) classifier to annotate taxonomic information for each representative sequence (James et al., 2014; Qiong et al., 2007). Based on OTU abundances and taxonomic annotation of OTUs, we obtained relative abundance profiles at the phylum, class, order, family, genus, and species levels. This made it easy to understand the overall pattern of each classification level annotated.

Phylogenetic relationships were used to reveal the differences between OTU sequences; combined with the species annotation information represented by each OTU sequence, we constructed a species classification tree. The dominant species were selected according to the results of the species classification.

\section{Species diversity analysis}

We calculated the values of sample alpha diversity indices by QIIME software, and the results were used to create the corresponding dilution curve. The dilution curve used the relative proportions of various known OTUs obtained from the 16S rRNA sequences to calculate the expected value of each alpha diversity index when extracting $n$ OTUs ( $n$ is less than the total number of measured reads sequences) and then based on a set of $n$ values (generally a set of equivariance series less than the total sequence number) and the expected value of the corresponding alpha diversity index. We prepared an alpha diversity index statistical table. In addition, the 16S rRNA gene amplicon sequence data were also analyzed based on weighted Unifrac distance, alpha diversity (Chao1 index, Shannon index, and Simpson index), and principal coordinates analysis $(\mathrm{PCoA})$.

\section{Isolation and identification of culturable Vibrio}

The 16S rRNA amplicon sequencing identified the genus Vibrio. To confirm the species, 36 samples from 2018 and 2019 were detected by isolation on CHROMagar Vibrio agar plates and identification using BD Phoenix-100. Then, the genes ompW, infC, $\operatorname{ctx} A, h l y A$, and $\operatorname{ch} x A$ et al. were detected by PCR (the primers and PCR conditions are listed in Table S1). V. cholerae isolates were subjected to $\mathrm{O} 1 / \mathrm{O} 139$ antigen serotyping using $V$. cholerae $\mathrm{O}$ antisera.

\section{Results}

\section{Quality of high-throughput sequencing profiles}

High-throughput amplicon sequencing from 36 samples yielded a total of 1, 839, 668 good-quality sequences. A total of 3, 883 OTUs were identified from nine migratory bird intestinal tracts, 13 migratory bird visceral organs, 3 migratory bird feces samples, 4 aquatic plants, and 7 water 
samples from 2018 (2, 518 OTUs) and 2019 (1, 365 OTUs) (annotation results of OTUs are listed in Table S2).

\section{Bacterial community composition}

The samples from 2018 to 2019 displayed different structures of gut microbiota. At the phylum level, Proteobacteria (45.17\%), Bacteroidetes (21.73\%), Firmicutes (20.25\%), Fusobacteria (9.96\%), and Verrucomicrobia (2.89\%) were the dominant bacterial phyla in 2018 (Figure 2). In 2019, Bacteroidetes, Firmicutes, Fusobacteria and Verrucomicrobia decreased to 3.37\%, 16.98\%, $0.10 \%$ and $0.5 \%$, respectively, and Proteobacteria increased to $54.90 \%$.

At the genus level, there were 20 genera with relative higher content in samples from 2018. These included Bacteroides (17.87\%), Clostridium (5.87\%), Plesiomonas (9.52\%), Vibrio (7.73\%), Fusobacterium (7.00\%), Aeromonas (6.26\%), Escherichia (4.76\%), Wohlfahrtiimonas (3.31\%), and Cetobacterium (2.95\%) (Figure 2) of the percentages of Aeromonas and Cetobacterium decreased to $0.40 \%$ and $0.10 \%$, respectively, and the percentage of others was less than $0.10 \%$ in 2019.

\section{Differences in species diversity}

To estimate the bacterial diversity in each sample, alpha diversity indices were calculated based on the OTUs. The Chao1 index varied from 102.200 to 966.074. The Shannon index varied from 0.803 to 7.379 , and the Simpson index varied from 0.142 to 0.986 in 2018. There were significant differences in alpha diversity indices between the samples in $2018(\mathrm{p}<0.05, t$ test).

The diversity, richness, and uniformity values of lake water were higher than those for aquatic plants and migratory bird remains. The Chao 1 index of $\mathrm{A} 3 \mathrm{C}$ was below those from the migratory bird remains. All indices of bird A19C were higher than those of other birds. However, there were no significant differences in alpha diversity indices between the three types of samples in 2019 $(\mathrm{p}>0.05, t$ test $)($ Table $\mathrm{S} 3)$.

According to the weighted Unifrac PCoA (Figure 3), the samples from 2018 were able to be clustered together except for four intestinal tract samples (A14C, A8C, A12C, A3C). Compared to 2018, the distributions of stool samples and environmental samples from 2019 were too scattered to be clustered. Only bird samples RS2.4 and RS3.1 could be clustered together.

\section{pH, salt content, total Vibrio amount, and total bacterial counts in the migratory bird habitat}

Table 2 shows $\mathrm{pH}$ values and salt contents during the sampling periods from 2018 to 2019 in Chifeng City, Inner Mongolia autonomous region, China. In 2018, the salt contents and amounts of Vibrio of Ganggeng Lake were lower than in Nanhekou and Beihekou. In 2019, the salt 
contents and amounts of Vibrio at these three sampling points had decreased compared to 2018.

\section{Confirmation of Vibrio genus}

Vibrio species were identified as $V$. alginolyticus, V. cholerae, and $V$. metschnikovii by $\mathrm{BD}$ Phoenix-100 testing. V. cholerae was detected through the omp $W$ gene in 2018, but Vibrio cholerae was not detected in 2019. All V. cholerae isolates harbored hlyA genes but not ctxA, tcpA, or $\operatorname{ch} x A$ genes. Additionally, all isolates were NOVC. By screening the infC gene, we also verified the presence of V. metschnikovii strains in samples from 2018 and 2019 (Table 3).

NOVC and $V$. metschnikovii were detected in six bird intestines; these two bacteria were only detected in bird NO. 5 and bird NO. 11 at the same time. This suggested that they had not spread throughout the bodies of the migratory birds. Interestingly, NOVC was present in the liver of bird No. 5; V. metschnikovii was present in the lungs of bird No. 11 and bird No. 13 but not in the intestines (Table 3). V. metschnikovii in the lungs could have been inhaled by migratory birds via the nasal cavity.

\section{Nucleotide Sequence}

\section{Accession Numbers}

The sequences of 16S rRNA amplicon were submitted to FigShare under the public site https://figshare.com/s/4ce5c0dd792898e1c32e.

\section{Discussion}

Dali Nouer Lake, Chifeng, China was named as one of the "Important Wetlands in Asia". It is an important migration channel for migratory birds in northern China, harboring 15 orders, 32 families, and 152 species birds. It also has had high yields of mandarin fish and crucian carp. Therefore, environmental changes should be monitored, especially the structure of the microbiota. At the phylum level, the relative abundance of Fusobacteria in dead migratory birds was higher than in healthy migratory birds from 2018 to 2019. Fusobacteria disturbed the intestinal microbiota balance via an increase in opportunistic pathogens and a decrease in probiotics $(\mathrm{Yu}$ Y.N., 2015). Fusobacteria was not the main phylum present in Dali Nouer Lake, and this indicated that these bacteria were carried by migratory birds. At the genus level, the relative abundances of Vibrio, Aeromonas, Plesiomonas, and Bacteroides were also higher in all samples in 2018. The relative abundances of these bacteria in healthy migratory birds had dropped in 2018 . The top 20 genera of the relative contents (except Vibrio and Aeromonas) in samples were mainly distributed in the migratory bird organs and intestines, and little was present in the environment. Therefore, it 
is possible these bacteria were carried by migratory birds.

It is worth noting that NOVC could simultaneously be isolated in water and dead migratory birds by PCR. O1/O139 V. cholerae that carries the cholera toxin has resulted in seven pandemics. The seventh $V$. cholerae pandemic continues to present day and has exhibited evolved characteristics compared to previous pandemics, rendering it difficult to treat cholera disease outbreaks (Hu D., 2016; Laviad-Shitrit S., 2018). Although NOVC isolates did not produce toxins that cause cholera, the possibility that NOVC can have other pathogenic characteristics cannot be overlooked. NOVC most commonly causes sporadic gastroenteritis, and less commonly is an invasive parenteral infection (Deshayes S., 2015). NOVC bacteremia is still rare, but it has been reported sporadically in some countries (Li X., 2020). The hemolysis and no CTX toxin characteristics of NOVC in the liver of bird No. 5 were consistent with the findings of George N et al. (2013). Thus, it is likely that migratory bird immunity had decreased due to the invasion of Fusobacteria, causing NOVC to invade the liver through the blood, not the intestines.

Fortunately, $V$. cholerae was not isolated and identified in the water or the feces of migratory birds in 2019. However, V. metschnikovii could be still isolated from the feces of migratory birds and from Nanhekou, Dali Nouer Lake. In contrast to V. cholerae, few reports have described the pathogenicity of V. metschnikovii. A cytolysin specific for V. metschnikovii with hemolytic properties was first described in 1981 (Miyake M, 1988). Although the pathogenicity of $V$. metschnikovii to migratory birds has been less frequently reported, it was also isolated in the feces of healthy migratory birds in 2019. Therefore, the bacteria possibly entered the bodies of migratory birds through feeding on contaminated food, and the bacteria did not cause harm to the migratory birds. The aquatic environment, as the main food source for migratory birds, plays an important role in the composition of the gut microbiota (Risely et al., 2018). The gut microbiota structure of migratory bird samples was similar to that of the environment in 2018, but not in 2019 (Figure 3). This suggests that the migratory birds from 2018 had been in contact with the lake for a period of time sufficient for their gut microbiota structure to have stabilized (Risely et al., 2018).

No avian influenza virus (AIV), Newcastle disease virus (NDV), avian infectious bursal disease virus (IBDV), avian infectious virus (IBV), avian infectious laryngotracheitis virus (ILTV), duck plague virus (DPV), gosling plague virus (GPV), avian paramyxovirus type 4 (apmv-4), or West Nile virus (WNV) were detected in dead migratory birds (data no shown).

\section{Conclusions}

By comprehensive analyses of physicochemical characteristics of Dali Nouer Lake and 16SrDNA of samples from 2018 and 2019, the microflora changes of healthy and dead birds caused by the 
$\mathrm{pH}$ changes of Dali Nouer Lake were analyzed. Migratory birds whose immune function had declined due to infection with Fusobacteria were infected by opportunistic pathogens. For several reasons, the salinity of Dali Nouer Lake has increased, making the area suitable for the proliferation and growth of bacteria from migratory birds. Therefore, long-term monitoring of the water quality in this migratory bird area is required.

\section{List of abbreviations}

PCR: Polymerase chain reaction; OTU: operational taxonomic unit; AIV: avian influenza viruses. NDV: new castle disease virus. IBDV: avian infectious bursal disease virus. IBV: avian infectious virus. ILTV: avian infectious laryngotracheitis virus. DPV: duck plague virus. GPV: gosling plague virus. APMV-4: avian paramyxovirus type 4. WNV: west nile virus. NOVC: non-O1/O139 V. cholerae.

\section{LITERATURE CIED}

Dijk JG, Verhagen JH, Wille M, Waldenström J. (2018). Host and virus ecology as determinants of influenza A virus transmission in wild birds. Curr.Opin.Virol. 28, 26-36.

Allarda SM, Callahan MT, Bui A, et al. (2019). Creek to Table: Tracking fecal indicator bacteria, bacterial pathogens, and total bacterial communities from irrigation water to kale and radish crops. Sci. Total Environ. 666, 461-471.

Alvarez P, Mattiello R, Rivailler P, et al. (2010). First isolation of an H1N1 avian influenza virus from wild terrestrial non-migratory birds in Argentina. Virology 396, 76-84.

Round, J. L., \& Mazmanian, S. K. (2009). The gut microbiota shapes intestinal immune responses during health and disease. Nature Reviews Immunology, 9, 313-323.

Sommer, F., \& Bäckhed, F. (2013). The gut microbiota-masters of host development and physiology. Nature Reviews Microbiology, 11, 227.

Zaneveld, J. R., McMinds, R., \& Vega, T. R. (2017). Stress and stability: Applying the Anna Karenina principle to animal microbiomes. Nature Microbiology, 2, 17121.

Verma S.K., Singh L. (2003). Novel universal primers establish identity of an enormous number of animal species for forensic application. Molecular Ecology Notes., 3:28-31.

Caporaso, J. G., Lauber, C. L., Walters, W. A., Berg-Lyons, D., Huntley, J., Fierer, N., ... Bauer, M. (2012). Ultra- high- throughput microbial community analysis on the Illumina HiSeq and MiSeq platforms. The ISME Journal, 6, 1621-1624.

James RC, Qiong W, Jordan A, et al. (2014). Ribosomal Database Project: data and tools for high 
throughput rRNA. analysis Nucl.Acids Res. 42, 633-642.

Qiong W, George M, James M, et al. (2007). Naïve Bayesian Classifier for Rapid Assignment of rRNA Sequences into the New Bacterial Taxonomy. Appl. Environ.Microbiol. 73, 5261-5267.

Yu YN, Yu TC, Zhao HJ, et al. (2015). Berberine may rescue Fusobacterium nucleatum-induced colorectal tumorigenesis by modulating the tumor microenvironment. Oncotarget. 6(31):32013-32026.

Hu D, Liu B, Feng L, Ding P, Guo X, Wang M, et al. (2016). Origins of the current seventh cholera pandemic. Proc Natl Acad Sci USA. 113:E7730-9.

Laviad-Shitrit S, Izhaki I, Arakawa E, Halpern M. (2018). Wild waterfowl as potential vectors of Vibrio cholerae and Aeromonas species. Trop Med Int Health. 23:758-64.

Deshayes S., Daurel C., Cattoir V., Parienti J.J., Quilici M.L., de La Blanchardière A. (2015). Non-O1, non-O139 Vibrio cholerae bacteraemia: case report and literature review. Springerplus. 4: 575.

Li X., Wu Y., Sun X., et al. (2020). Non-O1/non-O139 Vibrio cholerae bacteraemia in mainland China from 2005 to 2019: clinical, epidemiological and genetic characteristics. Epidemiol Infect. 148:e186.

Páll E., Niculae M., Brudașcă G. F., et al. (2021). Assessment and Antibiotic Resistance Profiling in Vibrio Species Isolated from Wild Birds Captured in Danube Delta Biosphere Reserve, Romania. Antibiotics, 10, 333.

Hirsch N., Kappe E., Gangl A., et al. (2020). Phenotypic and Genotypic Properties of Vibrio cholerae non-O1, non-O139 Isolates Recovered from Domestic Ducks in Germany. Microorganisms. 8, 1104.

Cardoso M. D., Lemos L. S., Roges E. M., et al. (2018). A comprehensive survey of Aeromonas $s p$. and Vibrio $s p$. in seabirds from southeastern Brazil: outcomes for public health. $J \mathrm{Appl}$ Microbiol. 124, 1283-1293.

George, N., Fredrick, F., Mohapatra, A., et al. (2013). Non-O1, non-O139 Vibrio cholerae sepsis in a patient with nephrotic syndrome. Indian journal of nephrology, 23, 378-380.

Johnson C. N. (2015). Influence of Environmental Factors on Vibrio spp. in Coastal Ecosystems. Microbiol Spectr. 3(3).

Miyake M., Honda T., Miwatani T. (1988). Purification and characterization of Vibrio metschnikovii cytolysin. Infect Immun. 56(4):954-60.

Risely A., Waite DW., Ujvari B., et al. (2018). Active migration is associated with specific and 294 


\section{Declarations}

\section{Ethics approval and consent to participate}

297 All migratory bird stool samples were collected under the supervision by the Wild Animal Sources

298 and Diseases Inspection Station, National Forestry and Grassland Bureau of China, and did not

299 cause any harm to the animals. All migratory bird epidemic material samples were provided by the local animal disease prevention and control center for bacteriological examination. The experimental protocol was established, according to the ethical guidelines of Helsinki Declaration and was approved by the Changchun Veterinary Research Institute, Chinese Academy of Agricultural Sciences (AMMS - 11 - 2020 - 11).

\section{Consent for publication}

Not Applicable.

\section{Availability of data and material}

All data generated or analysed during this study are available from the corresponding author on reasonable request.

\section{Competing interests}

313 The authors declare that they have no competing interests.

\section{Funding}

316 Funding for study design, data collection and data generation was provided by the National

317 Science and Technology Major Project of China (Grant agreement 2018ZX10733402). Publication

318 costs are also funded by the National Key Research and Development Programme of China 319 (NO.2016YFD0501305).

\section{Authors' contributions} PC, XJG conceived, directed, and carried out the study. LWZ, DC, LHX, JJ, YS and GJL prepared samples for sequence analysis; XJ, JYG, MWL and LZ acquired samples and analyzed the data. All authors have read and approved the final manuscript.

\section{Acknowledgements}

We are grateful to all members of Dali Nuoer Lake protected area, Chifeng city, Inner Mongolia autonomous region, China, which help for sampling. 


\section{$330 \quad$ Figure Legends}

331 Figure 1 Sample collection diagram. The green marks indicate the water sample collection 332 locations in 2018 and 2019.

333 Figure 2 Species classification and the relationship between different groups in 2018.

334 Different color sectors indicated different samples. The size of the sector indicated the relative

335 abundance ratio of the samples in the classification; the numbers below the classification name 336 indicated the relative abundance percentage.

337 Figure 3 Weighted Unifrac PCoA. H, B, S, and C represent aquatic plants, organ microbiota, 338 water planktonic flora, gut microbiota, respectively, from 2018. RS1, RS2, and RS3 represent 339 feces planktonic flora, water planktonic flora, and aquatic plants planktonic flora, respectively, 340 from 2019. 
Figures

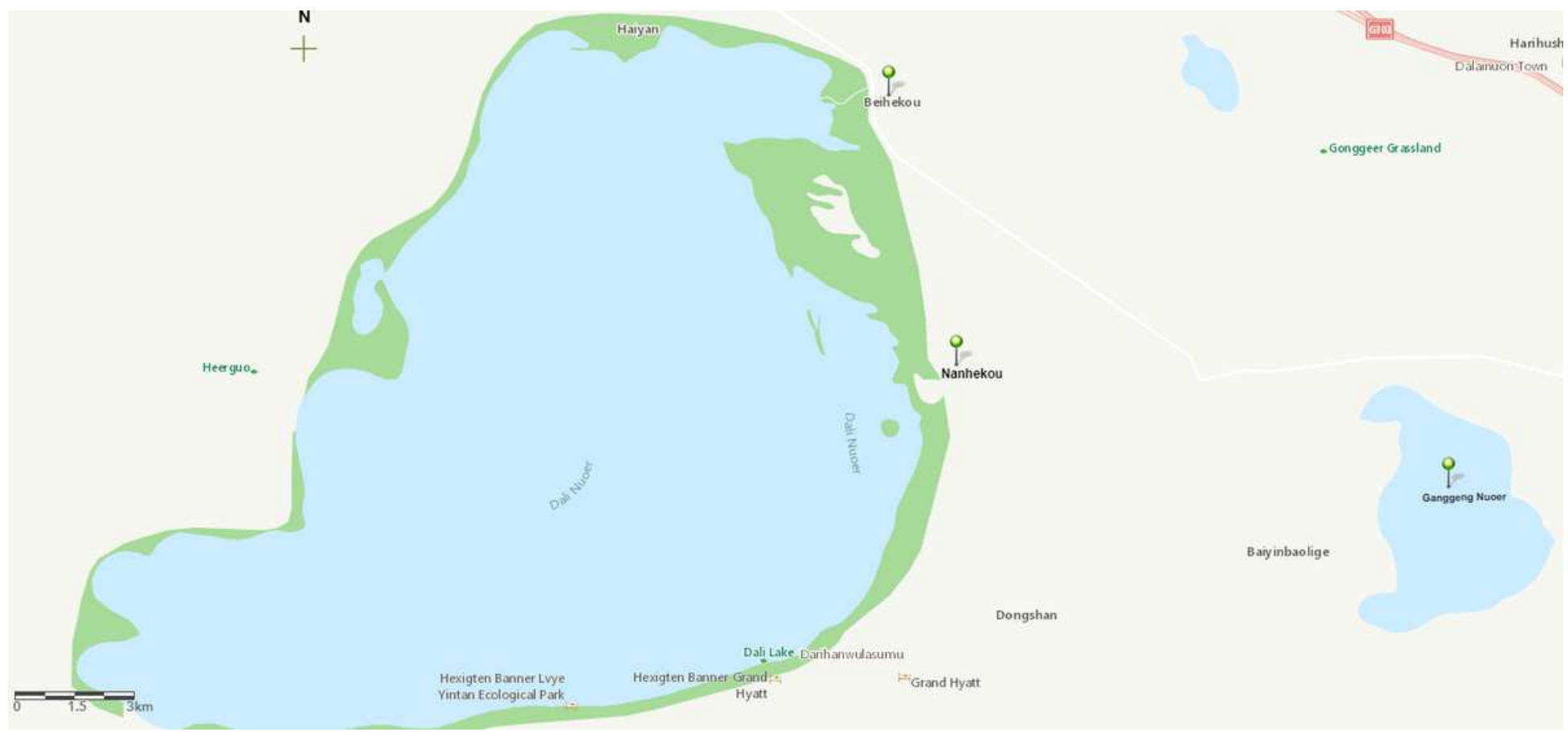

\section{Figure 1}

Sample collection diagram. The green marks indicate the water sample collection locations in 2018 and 201 


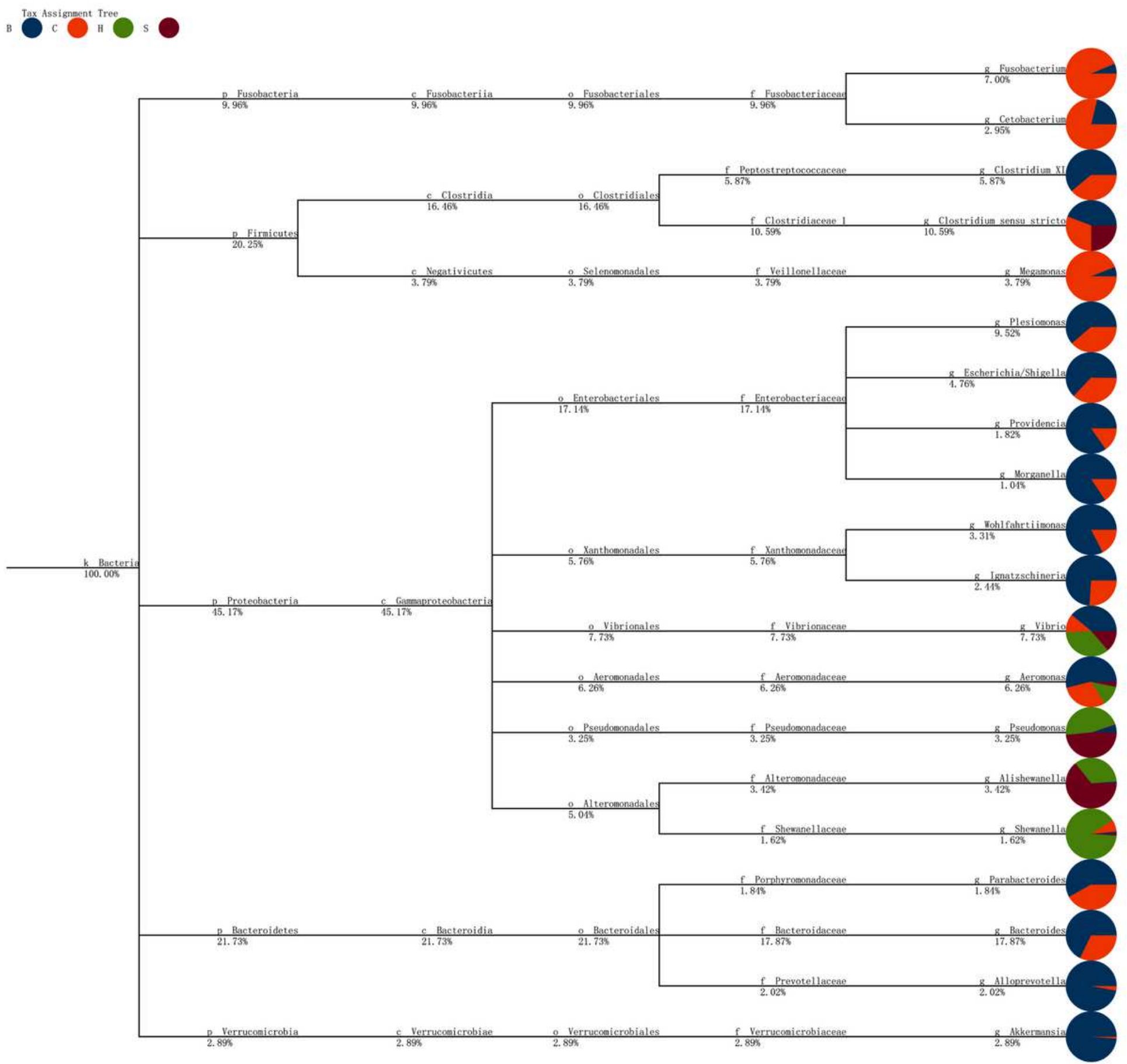

\section{Figure 2}

Species classification and the relationship between different groups in 2018. Different color sectors indicated different samples. The size of the sector indicated the relative abundance ratio of the samples in the classification; the numbers below the classification name indicated the relative abundance percentage 

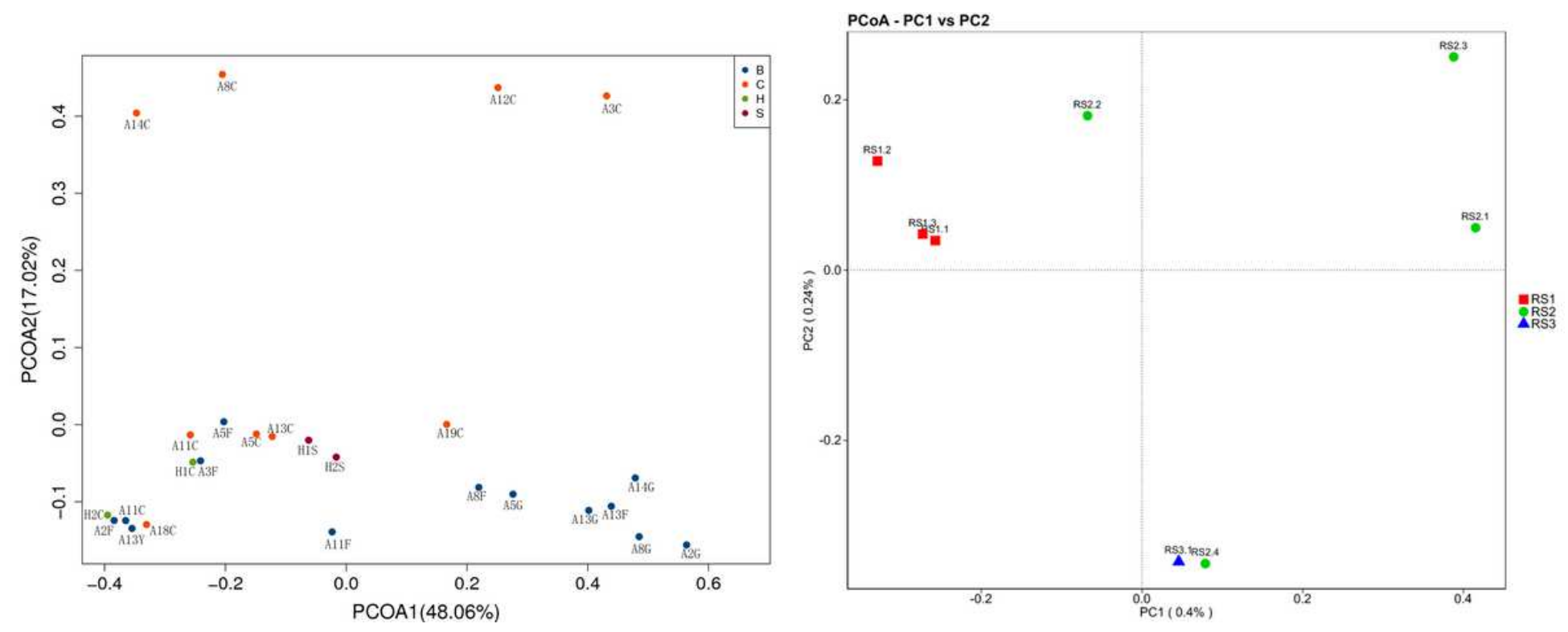

Figure 3

Weighted Unifrac PCoA. H, B, S, and C represent aquatic plants, organ microbiota, water planktonic flora, gut microbiota, respectively, from 2018. RS1, RS2, and RS3 represent feces planktonic flora, water planktonic flora, and aquatic plants planktonic flora, respectively, from 2019

\section{Supplementary Files}

This is a list of supplementary files associated with this preprint. Click to download.

- Table1.pdf

- Table2.pdf

- Table3.pdf

- SupplementTableS1/dentificationprimersusedinthisstudy.xls

- SupplementTables20TUsspeciesclassificcation.xls

- SupplementTableS3Alphadiversityindex.xls 